\title{
ASPECTOS BIOLÓGICOS E DANO DE Diatraea saccharalis (Fabr., 1794) (LEPIDOPTERA: PYRALIDAE) EM SORGO CULTIVADO SOB DIFERENTES DOSES DE NITROGÊNIO E POTÁSSIO
}

\author{
Biological aspects and damage of Diatraea saccharalis (Lepidoptera: Pyralidae) in sorghum, under \\ different doses of nitrogen and potassium
}

\author{
Sergio Antônio de Bortoli ${ }^{1}$, Háyda Oliveira Souza Dória ${ }^{2}$, \\ Nuno Miguel Mendes Soares Albergaria ${ }^{3}$, Maurício Vladimir Botti ${ }^{4}$
}

\section{RESUMO}

O presente trabalho teve como objetivo avaliar a influência da adubação da cultura do sorgo Sorghum bicolor (L.) Moench, na biologia da broca da cana-de-açúcar Diatraea saccharalis (Fabr., 1794) (Lepidoptera: Pyralidae). Para isso foi utilizado sorgo da cultivar Rubi-Asgrow, plantado em vasos utilizando-se diferentes doses de fertilizantes e mantidos em casa-de-vegetação. Os tratamentos utilizados (doses de NK) foram: N1 = 0-200 ppm; N2 = 50-200 ppm; N3 = 100-200 ppm; N4 = 200-200 ppm; N5 = 400-200 ppm; K1 = 200-0 ppm; K2 = 200-50 ppm; K3 = 200-100 ppm; K4 = 200-200 ppm; e K5 $=200-400 \mathrm{ppm}$. De modo geral, pode-se concluir que doses de 50 a $200 \mathrm{ppm}$ de $\mathrm{N}$ promoveram o desenvolvimento normal das larvas de $D$. saccharalis, sendo que as menores porcentagens de dano foram verificadas nas menores doses; para o potássio, quanto maior a dose, menor foi o dano causado pelas lagartas, apesar de favorecer o desenvolvimento da mesma.

Termos para indexação: broca-da-cana, Sorghum bicolor, biologia, insecta.

\section{ABSTRACT}

This work was carried out to evaluate the influence of the fertilization of sorghum, Sorghum bicolor (L.) Moench, on the stem borer Diatraea saccharalis (Fabr., 1794) (Lepidoptera: Pyralidae) biology. It was used the variety Rubi-Asgrow. The treatments were (NK doses): N1 = 0-200 ppm; N2 = 50-200 ppm; N3 = 100-200 ppm; N4 = 200-200 ppm; N5 = 400-200 ppm; $\mathrm{K} 1=200-0 \mathrm{ppm} ; \mathrm{K} 2=200-50 \mathrm{ppm} ; \mathrm{K} 3=200-100 \mathrm{ppm} ; \mathrm{K} 4=200-200 \mathrm{ppm}$ and K5 $=200-400 \mathrm{ppm}$. It was possible to conclude that nitrogen doses from 50 to $200 \mathrm{ppm}$ provide a normal development for $D$. saccharalis larvae, although the lowest percentage of damage was verified with the lowest dose; while for the potassium, the highest dose favoured the caterpillars development, but less damage was observed on the plants.

Index terms: sugarcane borer, Sorghum bicolor, biology, insecta.

(Recebido para publicação em 14 de Janeiro de 2004 e aprovado em 3 de agosto de 2004)

\section{INTRODUÇÃO}

O sorgo, Sorghum bicolor (L.) Moench, é uma espécie de origem tropical que hoje se encontra cultivada em quase todas as partes do mundo. Seus diversos tipos, com sua ampla variabilidade genética são usados principalmente como: cereal, forragem e cultura açucareira. Esta gramínea, pela sua rusticidade, resiste a condições adversas, como períodos secos e solos pobres (BOIÇA JÚNIOR \& LARA, 1983), porém apresenta uma série de problemas relativos à incidência de pragas, que podem comprometer a sua produção. Entre os insetos mais prejudiciais, está a Diatraea saccharalis (Fabr., 1794) (Lepidoptera: Pyralidae), que devido a grande expansão da cultura da cana-de-açúcar, também hospedeira dessa praga, passou a atacar com maior intensidade o sorgo (BOIÇA JÚNIOR \& LARA, 1993). Na região de Jaboticabal, SP, os danos podem chegar a 33,25\% da produção de grãos (LARA et al., 1980).

O dano provocado pela lagarta de $D$. saccharalis pode ser direto, por meio de abertura de galerias no interior do colmo da planta, reduzindo o fluxo de seiva, além de torná-la mais suscetível ao tombamento pela ação do vento e chuvas; ou indireto, quando os orifícios favorecem a penetração de microrganismos fitopatogênicos no interior do colmo (GALLO et al., 2002).

\footnotetext{
1. Prof. Dr. do Departamento de Fitossanidade - Faculdade de Ciências Agrárias e Veterinárias/UNESP - Via de Acesso Prof. Paulo Donato Castellane, s/n, 14884-900, Jaboticabal, SP - bortoli@fcav.unesp.br

2. Aluna do Doutorado do Deptartamento de Biologia , Faculdade de Filosofia, Ciências e Letras de Ribeirão Preto - Av. Bandeirantes, 3900 - $14040-901$ Ribeirão Preto, SP.

3. Aluno do Doutorado do Departamento de Fitossanidade, Faculdade Ciências Agrárias e Veterinárias, UNESP - Jaboticabal, SP.

4. Ex-Aluno do Departamento de Biologia, Faculdade de Filosofia, Ciências e Letras de Ribeirão Preto.
} 
Uma vez no interior do colmo, o controle das larvas torna-se muito difícil, sendo que, devido a tal dificuldade, passou-se a dar maior ênfase a trabalhos que busquem obter medidas alternativas, dentre elas a resistência de plantas, podendo ela ser constitutiva ou induzida (LARA, 1991).

De acordo com Matson (1980), a adubação nitrogenada aumenta a atividade fotossintética da planta e estimula a divisão celular, determinando aumento no teor de proteínas e na biomassa total. Por outro lado, a adubação nitrogenada provoca alterações na quantidade e qualidade do nitrogênio presente na planta, uma vez que aumenta os níveis de $\mathrm{N}$ solúvel, principalmente como aminoácidos livres, os quais podem ser assimilados por diversas espécies de insetos. Além disso, a fonte de fertilizante pode, também, influir na qualidade e quantidade de compostos solúveis de nitrogênio, sendo que os amoniacais levam a um maior acúmulo, quando comparados com os nítricos.

Trabalhos realizados com diferentes doses de nitrogênio, em várias culturas, mostram que muitas vezes o incremento da dose corresponde a um aumento na infestação das pragas, existindo alguns, porém, que mostram efeito contrário. Assim, Singh \& Singh (1977) verificaram que a incidência de brocas em arroz aumentava com o aumento do nitrogênio aplicado no solo, o mesmo acontecendo com Chilo zonellus (Swinhoe, 1884) (Lep.: Pyralidae) em milho (ZAAZOU et al., 1970).

Outros trabalhos como os de Teran (1979), com várias espécies de Diatraea (Lep.: Pyralidae) em canade-açúcar; de Zing et al. (1982), com Heliothis armigera (Hüebner, 1808) (Lep.: Noctuidae) em algodoeiro; de Taneja \& Dhindwal (1982), com Pectinophora gossypiella (Saund., 1844) (Lep.: Gelechiidae) em algodoeiro; e de Al-Zubaid \& Capinera (1983), com Spodoptera exígua (Hüebner, 1808)) (Lep.: Noctuidae) em diversas culturas, mostram que a elevação na dose de nitrogênio normalmente provoca aumento na população da praga com conseqüente elevação no prejuízo.

Por outro lado, Coutinho et al. (1981), comparando o efeito da adubação com NPK e calcário sobre a ocorrência de $D$. saccharalis em sorgo sacarino, verificaram que as maiores intensidades de infestações foram obtidas nas menores doses de nitrogênio. Ainda neste sentido, Moore \& Clements (1984), estudando a influência de adubos nitrogenados aplicados em um gramado de Lolium perenne L., constataram que as larvas de Oscinella vastator (Curtis, 1845) (Diptera: Chloropidae) foram mais abundantes nas parcelas que receberam baixas doses de nitrogênio.

Beringer \& Trolldenier (1978) consideraram que o nitrogênio e o potássio são os mais importantes nutri- entes envolvidos na "saúde" das plantas cultivadas e relatam que, de maneira geral, o excesso de nitrogênio provoca um aumento na suscetibilidade das plantas ao ataque de pragas e doenças, enquanto que o de potássio diminui. Segundo esses autores, a nutrição adequada das plantas com o potássio favorece a síntese de proteínas e reduz o acúmulo de carboidratos e compostos solúveis de nitrogênio nas células, situação esta que favorece diversas espécies de insetos e ácaros.

Tingey \& Singh (1980) salientam que o excesso de nitrogênio e as deficiências de potássio e fósforo normalmente determinam um maior acúmulo de nitrogênio solúvel nos tecidos das plantas, devido à inibição de proteínas na taxa de proteólise. Ainda, as alterações de ordem quantitativa e qualitativa no teor de nitrogênio solúvel das plantas, advindas das interações entre os diferentes nutrientes, influenciam de maneira expressiva a capacidade de desenvolvimento e sobrevivência de diversos artrópodos fitófagos.

No caso de vários nutrientes, entre eles nitrogênio e potássio, ocorre certa controvérsia nos resultados, pois alguns autores evidenciam influência negativa deles sobre as populações das pragas, enquanto que outros mostram efeitos positivos, e alguns descrevem casos onde não existe influência alguma (DE BORTOLI \& MAIA, 1994).

Ainda quanto ao potássio, Chaboussou (1999) cita ser um elemento essencial ao desenvolvimento e metabolismo das plantas, uma vez que tem participação ativa em processos de fundamental importância como os da biossíntese, fosforilação e síntese de ATP, ativação enzimática e síntese de proteínas. Relaciona-se também com a migração de aminoácidos livres e com sua utilização adequada na síntese de proteínas.

De maneira inversa aos adubos nitrogenados, os potássicos, de acordo com a maioria dos autores, conferem às plantas maior resistência às pragas. Contudo, da mesma forma que ocorre com o nitrogênio, alguns trabalhos mostram o efeito inverso gerando com isso alguma controvérsia.

Poucos são os trabalhos que estudam o efeito isolado do potássio sobre a incidência de pragas, estando quase sempre associado à outros elementos, principalmente ao nitrogênio e ao fósforo. Assim, Rahier (1979) relata que baixos teores de potássio favorecem o crescimento populacional de Myzus persicae (Sulzer, 1776) (Hem.: Aphididae) em nabo. Por outro lado, Carvalho et al. (1984) constataram que a falta do potássio na adubação do milho tornou a planta mais suscetível ao ataque de Spodoptera frugiperda (J.E. Smith, 1797) (Lep.: Noctuidae). 
A adubação também pode afetar outras características das plantas, como o teor de carboidratos, componentes fundamentais da dieta dos insetos (HAGEN et al., 1984). Um outro componente que também pode ser afetado pela adubação é o teor de tanino, sendo o sorgo uma espécie que apresenta altos teores de taninos em sua constituição, compostos que, normalmente, podem promover efeitos negativos sobre os animais, particularmente no comportamento alimentar de alguns insetos.

Neste sentido, conduziu-se este trabalho com objetivo de analisar a influência de diferentes doses de nitrogênio e potássio aplicados ao sorgo, sobre alguns aspectos biológicos de $D$. saccharalis e seus danos na citada cultura.

\section{MATERIAL E MÉTODOS}

O experimento foi conduzido no Departamento de Fitossanidade da FCAV-UNESP, Jaboticabal, SP, sendo o sorgo, da cultivar Rubi-Asgrow, cultivado em vasos de 10 litros, em casa-de-vegetação. O solo utilizado foi o Latossolo Vermelho Escuro - textura média, retirado da camada arável $(0-20 \mathrm{~cm})$, utilizando-se na adubação nitrogênio (sulfato de amônio - 21\% de N), fósforo (superfosfato simples - 18\% $\mathrm{P}_{2} \mathrm{O}_{5}$ ) e potássio (cloreto de potássio - 60\% de $\mathrm{K}_{2} \mathrm{O}$ ), sendo que em todos os vasos foram aplicados 0,125 ppm de bóro (Bórax) e 0,250 ppm de zinco (sulfato de zinco). Os tratamentos utilizados (doses de NK) foram: $\mathrm{N} 1=0$ 200 ppm; N2 = 50-200 ppm; N3 = 100-200 ppm; N4 = 200-200 ppm; N5 = 400-200 ppm; K1 = 200-0 ppm; K2 $=200-50 \mathrm{ppm} ; \mathrm{K} 3=200-100 \mathrm{ppm} ; \mathrm{K} 4=200-200$ ppm; e K5 = 200-400 ppm, sendo também aplicada a dose de 200 ppm de P para todos os tratamentos.

As doses de nitrogênio foram parceladas, sendo 1/3 aplicado no plantio e 2/3 em cobertura, 20 dias após a germinação. As doses de potássio foram aplicadas de uma única vez, por ocasião do plantio. Foram montados 200 vasos (20/tratamento), nos quais foram conduzidas 5 plantas por vaso, após o desbaste realizado 15 dias após a germinação.

No $30^{\circ}$ dia após o plantio, cada uma das plantas (30/tratamento) foi infestada com 15 lagartas recém eclodidas de $D$. saccharalis, sendo as mesmas colocadas na inserção da terceira folha a contar da extremidade de crescimento da planta. Aos 55 dias após a infestação, as plantas foram retiradas dos vasos com o sistema radicular, sendo os seus colmos abertos longitudinalmente, avaliando-se o número de internódios totais e broqueados e o comprimento total das galerias. As lagartas encontradas durante a amostragem foram indivi- dualizadas e submetidas à medições de comprimento, largura de tórax e peso. Posteriormente acompanhou-se o desenvolvimento das mesmas, recebendo como alimento pedaços de colmos do tratamento correspondente, até a passagem para o estágio pupal, obtendo-se assim o período larval, bem como o comprimento, largura e peso das pupas.

A determinação de nitrogênio nas plantas foi realizada segundo o método da digestão sulfúrica; de fósforo e potássio pela digestão nitro-perclórica (MALAVOLTA et al., 1997); de carboidratos solúveis pelo método descrito por Silva \& Queiroz (2002) e de taninos pelo método colorimétrico descrito por Rodrigues (1991).

As análises estatísticas das variáveis biológicas e de dano foram feitas utilizando-se o teste $\mathrm{F}$ em delineamento inteiramente casualizado, sendo as médias comparadas pelo teste de Tukey a 5\%, enquanto que para os componentes das análises químicas das plantas e parâmetros biológicos avaliados para as larvas, aplicou-se o teste de regressão múltipla.

\section{RESULTADOS E DISCUSSÃO}

As médias relativas ao comprimento, largura, peso e período larval de Diatraea saccharalis em sorgo cultivado em diferentes doses de nitrogênio encontramse na Tabela 1 . De acordo com esses dados nota-se que as doses de 50, 100 e $200 \mathrm{ppm}$ de $\mathrm{N}$ proporcionaram os maiores valores para comprimento de larvas $(17,10$; 17,75 e 19,00 mm), sendo que nas doses extremas (0 e $400 \mathrm{ppm}$ ) as lagartas apresentaram-se com tamanhos menores, evidenciando que o componente pode ser positivo ao desenvolvimento do inseto, sendo que na sua ausência ou doses excessivas podem atuar negativamente. A mesma situação é observada em relação ao peso das lagartas, sendo que quando se analisa a largura das larvas resultados estatisticamente semelhantes foram encontrados para todos os tratamentos. Apesar da não diferença significativa, nota-se uma certa tendência de a ausência de nitrogênio prolongar o período larval, enquanto que com o aumento da dose o período larval vai gradativamente reduzindo.

Analisando-se os dados relativos ao comprimento, largura, peso e período pupal para machos e fêmeas de $D$. saccharalis notou-se a não significância para todas as variáveis e tratamentos. Salienta-se que as doses extremas (0 e $400 \mathrm{ppm}$ ) mostram também a tendência em reduzir o tamanho das pupas, especialmente a dose máxima, sendo o período pupal também ligeiramente superior na dose mais elevada de nitrogênio. 
TABELA 1 - Comprimento, largura do tórax, peso e período larval de Diatraea saccharalis em sorgo cultivado em diferentes doses de nitrogênio, FCAV/UNESP, Jaboticabal, 2004.

\begin{tabular}{ccccc}
\hline $\begin{array}{c}\text { Tratamentos } \\
\text { (doses de NK em ppm) }\end{array}$ & $\begin{array}{c}\text { Comprimento de } \\
\text { Larva (mm) }\end{array}$ & $\begin{array}{c}\text { Largura doTórax } \\
\mathbf{( m m )}\end{array}$ & $\begin{array}{c}\text { Peso Larval } \\
\mathbf{( m g )}\end{array}$ & $\begin{array}{c}\text { Período Larval } \\
\text { (dias) }\end{array}$ \\
\hline N1 - (0-200) & $14,25 \mathrm{~b}$ & $2,13 \mathrm{a}$ & $54,40 \mathrm{~b}$ & $91,62 \mathrm{a}$ \\
N2 - (50-200) & $17,10 \mathrm{a}$ & $2,35 \mathrm{a}$ & $75,30 \mathrm{ab}$ & $84,27 \mathrm{a}$ \\
N3 - (100-200) & $17,75 \mathrm{a}$ & $2,20 \mathrm{a}$ & $78,64 \mathrm{ab}$ & $87,25 \mathrm{a}$ \\
N4 - (200-200) & $19,00 \mathrm{a}$ & $2,36 \mathrm{a}$ & $90,92 \mathrm{a}$ & $84,07 \mathrm{a}$ \\
N5 - (400-200) & $15,84 \mathrm{~b}$ & $2,22 \mathrm{a}$ & $65,90 \mathrm{ab}$ & $72,33 \mathrm{a}$ \\
\hline
\end{tabular}

Médias seguidas da mesma letra, nas colunas, não diferem significativamente entre si pelo teste de Tukey ao nível de $5 \%$ de probabilidade.

Na Tabela 2 estão apresentadas às médias da porcentagem de plantas atacadas e comprimento das galerias causadas pelas larvas de $D$. saccharalis avaliados. Nas doses de 100 e 200 ppm de $\mathrm{N}$ as parcelas apresentaram menores índices de infestação, sugerindo que nessas concentrações, favorecem a planta.

Na Tabela 3 encontram-se os resultados das análises químicas das plantas, observando-se que o aumento na dose de $\mathrm{N}$ na adubação do sorgo causa um aumento significativo na porcentagem de $\mathrm{N}$ e de taninos na planta, enquanto que a porcentagem de carboidratos decresce; as concentrações de $\mathrm{P}$ e $\mathrm{K}$ mostram-se pouco influenciadas pela alteração na quantidade de nitrogênio aplicada na adubação.

Apesar dessas diferenças, as análises de correlação entre os resultados da composição química do sorgo sob diferentes níveis de $\mathrm{N}$ na adubação e os parâmetros biológicos de $D$. saccharalis analisados, não mostraram qualquer tipo de correlação significativa.

Os resultados das observações no comprimento, largura, peso e período larval de $D$. saccharalis em sorgo cultivado em diferentes doses de potássio encontram-se na Tabela 4. Nota-se que as doses de 100, 200 e $400 \mathrm{ppm}$ de $\mathrm{K}$ proporcionaram os maiores valores para comprimento de larvas (19,13; 19,00; e 20,23 mm), sugerindo que esse elemento parece ser positivo ao desenvolvimento do inseto. A mesma situação é observada em relação ao peso das lagartas, sendo que quando se analisa a largura das larvas, resultados estatisticamente semelhantes foram encontrados para todos os tratamentos. Quanto ao período larval, a dose de $100 \mathrm{ppm}$ foi a que propiciou o menor valor, observando-se a tendência das doses menores e das mais elevadas alongarem o período de desenvolvimento das lagartas. De Bortoli et al. (2003) verificaram uma mortalidade de $100 \%$ das larvas na dose de $100 \mathrm{ppm}$, e sendo as outras doses $(0,50$, 200, 400 ppm) estatisticamente iguais.

Na Tabela 5 encontram-se os resultados dos danos causados às plantas pelas larvas de $D$. saccharalis, avaliados pela porcentagem de plantas atacadas e comprimento total de galerias causadas pela larva. As plantas apresentaram menores índices de infestação na ausência de $K$, sugerindo que a ausência do potássio torna o sorgo menos suscetível ao ataque de $D$. saccharalis.

Na Tabela 6 observa-se que nos resultados das análises químicas das plantas, as porcentagens de carboidratos, nitrogênio, fósforo e taninos mostram-se pouco influenciadas pela alteração na quantidade de potássio aplicada na adubação. Apesar das diferenças nas concentrações de potássio, as análises de correlação entre os resultados das análises químicas do sorgo sob diferentes níveis de K na adubação e os parâmetros biológicos de $D$. saccharalis analisados, não mostraram qualquer tipo de correlação significativa. 
TABELA 2 - Médias da porcentagem de plantas atacadas e do comprimento das galerias provocadas por Diatraea saccharalis em sorgo cultivado em diferentes doses de nitrogênio, FCAV/UNESP, Jaboticabal, 2004.

\begin{tabular}{lcc}
\hline $\begin{array}{c}\text { Tratamentos } \\
\text { (doses de NK em ppm) }\end{array}$ & Planta atacada (\%) & Comprimento da galeria (cm) \\
\hline N1 - (0-200) & $6,36 \mathrm{c}$ & $19,63 \mathrm{ab}$ \\
N2 - (50-200) & $70,53 \mathrm{a}$ & $23,55 \mathrm{a}$ \\
N3 - (100-200) & $57,41 \mathrm{ab}$ & $14,45 \mathrm{bc}$ \\
N4 - (200-200) & $49,70 \mathrm{~b}$ & $14,39 \mathrm{bc}$ \\
N5 - (400-200) & $65,47 \mathrm{a}$ & $12,52 \mathrm{c}$ \\
\hline
\end{tabular}

Médias seguidas da mesma letra, nas colunas, não diferem significativamente entre si pelo teste de Tukey ao nível de $5 \%$ de probabilidade.

TABELA 3 - Valores de N, K, carboidratos e taninos solúveis em colmos de sorgo cultivado em diferentes doses de nitrogênio, FCAV/UNESP, Jaboticabal, 2004.

\begin{tabular}{lccccc}
\hline $\begin{array}{c}\text { Tratamentos } \\
\text { (doses de NK em ppm) }\end{array}$ & $\begin{array}{c}\text { Nitrogênio } \\
\mathbf{( \% )}\end{array}$ & Fósforo (\%) & Potássio (\%) & $\begin{array}{c}\text { Carboidratos } \\
\mathbf{( \% )}\end{array}$ & Taninos (\%) \\
\hline N1 - (0-200) & 0,46 & 0,08 & 1,55 & 10,20 & 9,33 \\
N2 - (50-200) & 0,61 & 0,09 & 1,99 & 9,33 & 13,13 \\
N3 - (100-200) & 1,00 & 0,13 & 1,93 & 7,77 & 12,53 \\
N4 - (200-200) & 1,35 & 0,13 & 1,66 & 8,27 & 19,16 \\
N5 - (400-200) & 1,80 & 0,10 & 1,61 & 6,09 & 18,13 \\
\hline
\end{tabular}

TABELA 4 - Comprimento, largura do tórax, peso e período larval de Diatraea saccharalis em sorgo cultivado em diferentes doses de potássio, FCAV/UNESP, Jaboticabal, 2004.

\begin{tabular}{lcccc}
\hline $\begin{array}{c}\text { Tratamentos } \\
\text { (doses de NK em ppm) }\end{array}$ & $\begin{array}{c}\text { Comprimento de } \\
\text { Larva (mm) }\end{array}$ & $\begin{array}{c}\text { Largura do } \\
\text { Tórax (mm) }\end{array}$ & $\begin{array}{c}\text { Peso Larval } \\
(\mathbf{m g})\end{array}$ & $\begin{array}{c}\text { Período Larval } \\
\text { (dias) }\end{array}$ \\
\hline K1 - (200-0) & $16,20 \mathrm{bc}$ & $2,07 \mathrm{a}$ & $62,98 \mathrm{~b}$ & $76,00 \mathrm{ab}$ \\
K2 - (200-50) & $14,50 \mathrm{c}$ & $2,14 \mathrm{a}$ & $60,50 \mathrm{~b}$ & $75,66 \mathrm{ab}$ \\
K3 - (200-100) & $19,13 \mathrm{ab}$ & $2,34 \mathrm{a}$ & $93,54 \mathrm{ab}$ & $68,83 \mathrm{~b}$ \\
K4 - (200-200) & $19,00 \mathrm{ab}$ & $2,36 \mathrm{a}$ & $90,92 \mathrm{ab}$ & $84,07 \mathrm{a}$ \\
K5 - (200-400) & $20,23 \mathrm{a}$ & $2,42 \mathrm{a}$ & $108,60 \mathrm{a}$ & $81,13 \mathrm{ab}$ \\
\hline
\end{tabular}

Médias seguidas da mesma letra, nas colunas, não diferem significativamente entre si pelo teste de Tukey ao nível de $5 \%$ de probabilidade. 
BORTOLI, S. A. de et al.

TABELA 5 - Porcentagens de plantas atacadas e comprimento total de galerias provocadas por Diatraea saccharalis em sorgo cultivado em diferentes doses de potássio, FCAV/UNESP, Jaboticabal, 2004.

\begin{tabular}{lcc}
\hline \multicolumn{1}{c}{$\begin{array}{c}\text { Tratamentos } \\
\text { (doses de NK em ppm) }\end{array}$} & Planta atacada (\%) & Comprimento da galeria (cm) \\
\hline K1 - (200-0) & $52,32^{\mathrm{a}}$ & $18,21 \mathrm{a}$ \\
K2 - (200-50) & $40,01 \mathrm{~b}$ & $7,63 \mathrm{~b}$ \\
K3 - (200-100) & $41,39 \mathrm{~b}$ & $9,60 \mathrm{~b}$ \\
K4 - (200-200) & $49,70 \mathrm{ab}$ & $14,38 \mathrm{ab}$ \\
K5 - (200-400) & $42,88 \mathrm{~b}$ & $9,78 \mathrm{~b}$ \\
\hline
\end{tabular}

Médias seguidas da mesma letra, nas colunas, não diferem significativamente entre si pelo teste de Tukey ao nível de $5 \%$ de probabilidade.

TABELA 6 - Valores de N, P, K, carboidratos e taninos solúveis em colmos de sorgo cultivado em diferentes doses de potássio, FCAV/UNESP, Jaboticabal, 2004.

\begin{tabular}{lccccc}
\hline $\begin{array}{c}\text { Tratamentos } \\
\text { (doses de NK em ppm) }\end{array}$ & $\begin{array}{c}\text { Nitrogênio } \\
\mathbf{( \% )}\end{array}$ & $\begin{array}{c}\text { Fósforo } \\
\mathbf{( \% )}\end{array}$ & Potássio (\%) & $\begin{array}{c}\text { Carboidratos } \\
\mathbf{( \% )}\end{array}$ & Taninos (\%) \\
\hline K1 - (200-0) & 1,15 & 0,12 & 0,60 & 5,78 & 13,83 \\
K2 - (200-50) & 1,27 & 0,14 & 0,92 & 7,71 & 15,60 \\
K3 - (200-100) & 1,33 & 0,11 & 1,43 & 7,09 & 14,50 \\
K4 - (200-200) & 1,35 & 0,13 & 1,66 & 8,27 & 19,16 \\
K5 - (200-400) & 1,18 & 0,13 & 2,02 & 6,59 & 14,13 \\
\hline
\end{tabular}

\section{CONCLUSÃO}

De modo geral, pode-se concluir que para o caso da adubação nitrogenada no sorgo, as doses mais equilibradas tendem a promover um bom desenvolvimento para as larvas de $D$. saccharalis, sendo que as menores porcentagens de dano foram verificadas nas menores doses de N (100, 200 e $400 \mathrm{ppm})$; enquanto que para o caso do potássio, quanto maior a dose, apesar de favorecer o desenvolvimento das lagartas, tornam as plantas menos danificadas pelo inseto.

\section{REFERÊNCIAS BIBLIOGRÁFICAS}

AL-ZUBAID, F. S.; CAPINERA, J. C. Aplication of different nitrogen levels to the most plant and cannibalistic behavior of beet armyworm, Spodoptera exigua (Hüebner) (Lepidoptera: Noctuidae). Environmental Entomology, College Park, v. 12, p. 1687-1689, 1983.
BERINGER, H.; TROODENIER, G. Influence of $\mathrm{K}$ nutrition on the response to environmental stress. In: CONGRESS IPI. POTASSIUM RESEARCH-REVIEW AND TRENDS, 11., 1978. Proceeding... [S.l.: s.n.], 1978. p. 189-222.

BOIÇA JÚNIOR, A. L.; LARA, F. M. Avaliação de resistência de genótipos de Sorghum bicolor (L.) Moench a Diatraea saccharalis (Fabricius, 1794) (Lepidoptera: Pyralidae) e determinação dos tipos envolvidos. Anais da Sociedade Entomológica do Brasil, Jaboticabal, v. 12, n. 2, p. 261-272, 1983.

BOIÇA JÚNIOR, A. L.; LARA, F. M. Resistência de genótipos de sorgo ao ataque de Diatraea saccharalis (Fabr.) (Lepidoptera: Pyralidae). Anais da Sociedade Entomológica do Brasil, Jaboticabal, v. 22, n. 2, p. 245-252, 1993. 
DE BORTOLI, S. A. de et al. Aspectos biológicos de Diatraea saccharalis (Fabr.) (Lepidoptera: Pyralidae) em Sorghum bicolor (L.) Moench sob diferentes níveis de potássio, em laboratório. Boletin de Sanidad Vegetal Plagas, Madrid, v. 29, n. 4, p. 575-580, 2003.

DE BORTOLI, S. A.; MAIA, I. G. Influência na aplicação de fertilizantes na ocorrência de pragas. In: SÁ, M. E. de; BUZZETI, S. Importância da adubação na qualidade dos produtos agrícolas. São Paulo: Ícone, 1994. p. 53-63.

CARVALHO, R. B. de et al. Estudo de diferentes dosagens de potássio em milho (Zea mays L.) influindo sobre Spodoptera frugiperda (J.E. Smith, 1797). Ecossistema, Espírito Santo do Pinhal, v. 9, p. 95-100, 1984.

CHABOUSSOU, F. Plantas doentes pelo uso de agrotóxicos: a teoria da trofobiose. 2. ed. Porto Alegre: L\&PM, 1999. 272 p.

COUTINHO, E. L. M. et al. Efeito do N, P, K e calcário sobre a incidência de Diatraea saccharalis (Fabr., 1794) (Lep.-Pyralidae) em sorgo sacarino. In: CONGRESSO BRASILEIRO DE ENTOMOLOGIA, 7., 1981, Fortaleza. Resumos... Fortaleza: SEB, 1981. p. 208.

GALLO, D. et al. Entomologia agrícola. Piracicaba: FEALQ, 2002. 920 p.

HAGEN, K. S.; DADD, R. H.; REESE, J. The food of insect. In: HUFFAKER, C. B.; RABB, R. L. Ecological Entomology. New York: John Willey \& Sons, 1984. p. 79-112.

LARA, F. M. Princípios de resistência de plantas a insetos. 2. ed. São Paulo: Ícone, 1991. 336 p.

LARA, F. M.; BARBOSA FILHO, G. C.; BARBOSA, J. C. Danos acarretados por Diatraea saccharalis (Fabr., 1794) na produção do sorgo granífero. Científica, São Paulo, v. 8, n. 1/2, p. 105-111, 1980.

MALAVOLTA, E.; VITTI, G. C.; OLIVEIRA, S. A. Avaliação do estado nutricional das plantas: princípios e aplicações. 2. ed. Piracicaba: POTAFOS, 1997. 319 p.

MATSON, W. J. Hebivory in relation to plant nitrogen content. Annual Review Of Ecology, Evolution, And Systematics, Palo Alto, v. 11, p. 119-161, 1980.
MOORE, D.; CLEMENTS, R. O. Stem boring diptera in perinnial eyegrass in relation to fertilizer. Annals Of Applied Biology, Warwick, v. 105, n. 1, p. 1-6, 1984.

RAHIER, H. Performance of Myzus persicae and production of its plant, Brassica rapa, related to plant mineral nutrition. Journal of Applied Entomology, Hamburg, v. 67, n. 9, p. 452, 1979.

RODRIGUES, W. A. Variabilidade para teor de tanino em sorgo (Sorghum bicolor) seu controle genético e associação com resistência a pássaros. 1991. 72 f. Dissertação (Mestrado em Genética e Melhoramento) - Universidade Federal de Lavras, Lavras, 1991.

SILVA, D. J.; QUEIROZ, A. C. Análise de alimentos: métodos químicos e biológicos. 3. ed. Viçosa: UFV, 2002. 235 p.

SINGH, R.; SINGH, M. P. Studies on varietal - cum manurial responses on the incidence of paddy stem borer (Tryporyza incertulas Wilk.). Madras Agricultural Journal, Coimbatore, v. 64, n. 4, p. 247-251, 1977.

TANEJA, S. L.; DHINDWAL, A. S. Bollworm incidence as affected by sowing date, nitrogen application and plant population in plant cotton. Indian Journal Plant Protection, New Delhi, v. 10, n. 1/2, p. 1-6, 1982.

TERAN, F. O. Sugarcane nutrition modifies infestation by Diatraea spp. Entomology Newsletter, [S.l.], v. 6, p. 20-23, 1979.

TINGEY, W. M.; SINGH, S. R. Environmental factors influencing the magnitude and expression of resistance. In: MAXWELL, F. G.; JENKINS, P. R. (Eds.). Breeding plants resistant to insects. New York: John Willey \& Sons, 1980. p. 87-113.

ZAAZOU, H. M.; EL-NAHAL, A. K. M.; BISHARA, M. A. The effect of certain cultural practices on the infestation of rice by Chilo agamennon Bles. and Tryporiza incertulas (Lepidoptera: Pyralidae). Bulletin De La Societe Entomologique D' Egypte, Le Caire, v. 54, p. 149-154, 1970.

ZING, Y. L.; GONG, P. Y.; JIANG, L. R. Effects of nitrogen fertilizer application of the cotton plant and the bollworm. Acta Entomologica Sinica, Peking, v. 25, n. 1, p. 16-23, 1982. 